\title{
The Effects of Buffer Molarity, Agitation Rate, and Mesh Size on Verapamil Release from Modified-Release Mini-Tablets Using USP Apparatus 3
}

\author{
Sandile M. M. Khamanga and Roderick B. Walker ${ }^{1}$ \\ Faculty of Pharmacy, Rhodes University, P.O. Box 94, Grahamstown, South Africa, 6140
}

\begin{abstract}
The effects of agitation rate, buffer molarity, and mesh size on the dissolution rate of verapamil hydrochloride from sustainedrelease matrix tablets were studied using USP Apparatus 3. Eudragit ${ }^{\circledR}$ and Carbopol ${ }^{\circledR}$ were used as rate-retarding polymers in tablets prepared by wet granulation. The study was conducted to determine whether the drugs exhibit similar release characteristics when tested under the same dissolution conditions. It was found that the dissolution rate of verapamil hydrochloride was affected by the variables assessed in these studies.
\end{abstract}

\section{Introduction}

$\mathrm{n}$ vitro dissolution testing is a critical component in the assessment of quality of a pharmaceutical product and

for the validation of the development and manufacturing process of a product (1). Dissolution testing is also used to assess the rate at which a drug is released from a dosage form, as it provides a manufacturer with a rational basis on which to predict in vivo behaviour and dosage form optimisation (2).

The granulation method used during manufacture may alter the rate of drug release from a formulation (3), as may the size, shape (4), and mechanical strength of a dosage form (5). Variables independent of dosage form design that may affect dissolution rates include temperature and $\mathrm{pH}$ of the dissolution media (3). The influence of temperature can be minimised in vitro by performing dissolution experiments at temperatures representative of the in vivo physiological conditions to which a dosage form would be exposed. In addition, agitation of the dissolution medium will influence the hydrodynamics of a test system, which in turn will affect the formation of a stagnant boundary layer, sink conditions, and consequently the rate of drug release from specific dosage forms $(6,7)$.

The purpose of these studies was to evaluate the effects of changing buffer molarity, agitation rate, and mesh/screen size on the release rate of a drug compound from sustainedrelease, hydrophilic matrix tablet formulations developed in our laboratory using USP Apparatus 3. Verapamil hydrochloride (VRP) was selected as a model drug, as it is well known and is widely used in the control of hypertension and angina.

\section{Materials and Methods}

\section{Materials}

Verapamil hydrochloride (Aspen-Pharmacare, Port Elizabeth, SA), Carbopol ${ }^{\circledR} 974$ PNF (Noveon, Inc., Brecksville,

${ }^{1}$ Corresponding author.

\begin{tabular}{|c|c|c|}
\hline & Ingredients & $\% w / w$ \\
\hline \multirow[t]{4}{*}{1.} & VRP & 33.0 \\
\hline & Carbopol ${ }^{\circledR}$ 974P NF & 5.0 \\
\hline & Eudragit ${ }^{\circledR} \mathrm{RS}$ & 7.5 \\
\hline & Emcocel $^{\circledR} 90 \mathrm{M}$ & 10.0 \\
\hline 2. & Surelease ${ }^{\circledR}$ E-7-19010 & 3.0 \\
\hline \multirow[t]{4}{*}{3.} & Carbopol ${ }^{\circledR}$ 974P NF & 5.0 \\
\hline & Eudragit ${ }^{\circledR} \mathrm{RS}$ & 6.0 \\
\hline & Emcocel ${ }^{\circledR} 90 \mathrm{M}$ & 10.0 \\
\hline & Emcompress ${ }^{\circledR}$ & 20.0 \\
\hline
\end{tabular}

4. Magnesium stearate $\quad 0.5$

Cleveland, USA), Eudragit ${ }^{\circledR}$ RS (Rohm Pharma Polymers, Darmstadt, GmBH), Emcompress ${ }^{\circledR}$ (Penwest Pharmaceutical Co., Mendel,UK), Emcoce ${ }^{\circledR}$ 90M (Penwest Pharmaceutical Co., Mendel, UK), Ethocel ${ }^{\circledR} 10$ FP (Dow Chemical Co., Michigan, USA), Surelease ${ }^{\circledR}$ E-7-19010 (Colorcon ${ }^{\circledR}$ LTD, Dartford, Kent, UK), and magnesium stearate (Aspen Pharmacare, Port-Elizabeth, SA) were used as received. Potassium hydrogen phosphate and o-phosphoric acid (85\%) were purchased from Merck Laboratories (Merck Chemicals Ltd, Midrand, South Africa).

\section{Methods}

Preparation of Tablets

Sustained-release VRP tablets were prepared using the formula listed in Table 1 by blending VRP, Carbopol ${ }^{\circledR}$ 974P $\mathrm{NF}$, Eudragit ${ }^{\circledR} \mathrm{RS}$, and Emcocel ${ }^{\circledR} 90 \mathrm{M}$. The powders were weighed separately using a top-loading electronic balance Model PM 4600 (Mettler, Zurich, Switzerland), screened (mesh screen no. 20), and granulated with Surelease ${ }^{\circledR}$ E-719010 using a Kenwood planetary mixer (Kenwood, UK) set at position 1. Prior to granulation, the Surelease ${ }^{\circledR}$ E-7-19010 
Table 2. Dissolution Test Conditions for USP Apparatus 3

\begin{tabular}{lccc}
\hline Parameter & \multicolumn{3}{c}{ Settings } \\
\hline Medium & $175 \mathrm{~mL}$ of $0.1 \mathrm{M}$ phosphate buffer \\
\hline & Row & $p H$ & Time in Medium (hours) \\
\cline { 3 - 4 } & & & \multicolumn{1}{c}{ VRP } \\
\hline 1 & 1.6 & 1 \\
2 & 3.4 & 4 \\
3 & 4.7 & 4 \\
4 & 6.8 & 4 \\
5 & 7.2 & 8 \\
\hline
\end{tabular}

Temperature $\quad 37 \pm 0.5^{\circ} \mathrm{C}$

dispersion was diluted to a $15 \% \mathrm{w} / \mathrm{v}$ solids content to reduce the viscosity and facilitate spraying of the granulation fluid onto the powder blend. The granules were then passed through a sieve (US sieve no. 20) using an oscillating granulator (Erweka, $\mathrm{GmbH}$, Germany) set at $50 \mathrm{rpm}$. The granules were dried in an oven maintained at $60^{\circ} \mathrm{C}$ for 12 hours, after which they were re-screened (US sieve no. 20). The weight of the granule mass was recorded. Carbopol ${ }^{\circledR}$ 974P NF, Eudragit ${ }^{\circledR}$ RS, Emcompress ${ }^{\circledR}$, and Emcoce ${ }^{\circledR} 90 \mathrm{M}$ were weighed, screened (mesh screen no. 20), and blended with the granules in a 1-kg capacity cube blender set at a horizontal angle. Blending was effected at $100 \mathrm{rpm}$ for 30 minutes to ensure adequate mixing. The mixture was lubricated with sieved magnesium stearate (US sieve no. 40) and added to the mixture, which was blended for an additional three minutes. Mini-tablets were prepared with 7-mm flatfaced punches using a Manesty ${ }^{\circledR} F 3$ single-punch tablet press (Manesty, Liverpool, England) and compressed to a target hardness of $90 \mathrm{~N}$. Tablets were de-dusted using a vacuum through a sieve and assessed within 48 hours of preparation.

Mini-tablets containing VRP were manufactured so as to contain $80 \mathrm{mg}$ of VRP in each unit. Three tablets were placed in a size 00 capsule to produce a dosage form containing $240 \mathrm{mg}$ VRP that could be compared to Isoptin ${ }^{\circledR}$ SR 240 -mg tablets.

\section{Drug Release Studies}

The in vitro dissolution profiles of the sustained-release matrix tablets were characterised using USP Apparatus 3 (VanKel Industries, New Jersey, USA). A model VK 750D digitally controlled water circulation/heater (VanKel Industries, New Jersey, USA) was used to maintain the temperature of the dissolution media at $37 \pm 0.5^{\circ} \mathrm{C}$. Samples were collected at predetermined time intervals, and a summary of the dissolution test conditions for these studies is depicted in Table 2. Samples were passed through 0.45- $\mu$ m Durapore ${ }^{\circledR}$ membrane HVLP filters (Millipore Corporation, Ireland) and

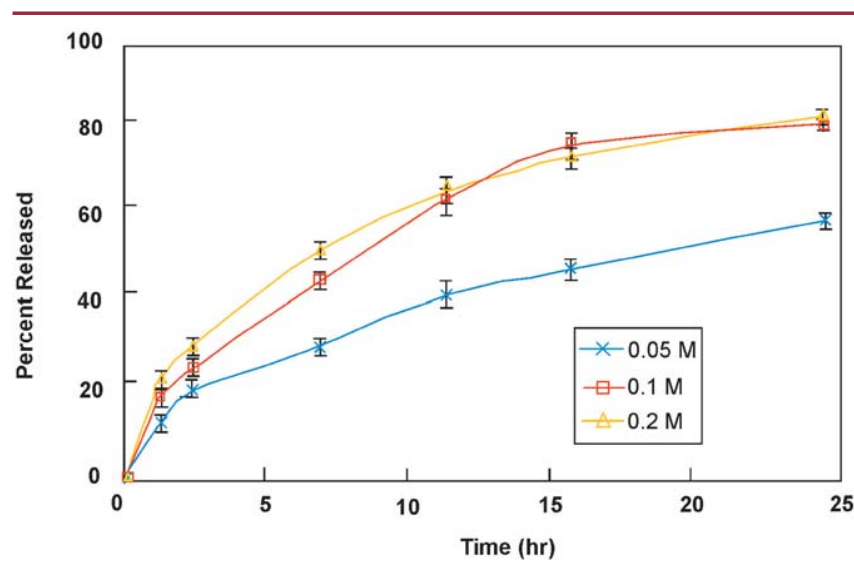

Figure 1. The effect of buffer molarity on VRP release from capsules containing hydrophilic matrix tablets (3) reported as mean $\pm S D(n=6)$.

analysed by a validated reversed-phase HPLC method developed in our laboratories (8).

The dosage forms were reciprocated sequentially in six dissolution vessels, and the agitation rates of 10,20 , and 30 dips per minute (dpm) were evaluated using $0.1 \mathrm{M}$ phosphate buffers. The effect of buffer molarity was also evaluated using buffers of $0.05,0.1$, and $0.2 \mathrm{M}$ at the $\mathrm{pH}$ values described in Table 2 at an agitation rate of $20 \mathrm{dpm}$. Six tablets were assessed for each condition; the resultant dissolution profiles were compared using the $f_{1}$ and $f_{2}$ difference and similarity factors as defined by Moore and Flanner (9).

\section{Results and Discussion}

Visual observation of the tablets during dissolution testing revealed that swelling was dominant during the test procedure. Swelling occurred in a predominantly axial direction, although some radial swelling was also observed, and these results are in agreement with previously reported results (10). The degree of swelling was significant when evaluating the dissolution of VRP tablets at a pH of 7.2 as opposed to a pH of 1.6. As hydration and swelling progressed, the VRP mini-tablets (3) that had been enclosed

Table 3. Results of Dissolution Profile Comparisons using the $f_{1}$ and $f_{2}$ Equations

\begin{tabular}{lc|c}
\hline Effect of Agitation Rate & & \\
\hline Conditions (dips/minute) & $\mathrm{f}_{1}$ & $\mathrm{f}_{2}$ \\
\hline 10 versus 20 & VRP & VRP \\
10 versus 30 & 60.0 & 36.0 \\
20 versus 30 & 87.7 & 24.8 \\
\hline Effect of Buffer Molarity & 29.5 & 41.9 \\
\hline 0.05 versus 0.1 & & \\
0.05 versus 0.2 & 50.7 & 37.3 \\
0.1 versus 0.2 & 60.5 & 35.3 \\
\hline
\end{tabular}


in the size 00 capsule formed a single rod-like cylinder, adhering to one another as the capsule disintegrated.

\section{Effect of Molarity}

The release profiles of VRP in media of different molarity are depicted in Figure 1. The calculated difference and similarity factors, $\mathrm{f}_{1}$ and $\mathrm{f}_{2}$, indicate that the VRP dissolution profile in the $0.05 \mathrm{M}$ buffer is different from the profiles in the $0.1 \mathrm{M}$ and $0.2 \mathrm{M}$ buffers. However, the calculated values indicate similarity between the profiles in buffers of $0.1 \mathrm{M}$ and $0.2 \mathrm{M}$ (Table 3 ). It is also evident that as the molarity of the dissolution medium increased from $0.05 \mathrm{M}$ to $0.2 \mathrm{M}$, the release rate of VRP increased as shown in Figure 1.

These results are in agreement with a report describing the effect of molarity on dissolution rates of chlorpheniramine maleate, another cationic drug, from beads manufactured by extrusion-spheronization (11). The high molarity of the dissolution medium is thought to weaken the $\mathrm{rigid} / \mathrm{cross}$-linked structure of carbomer gels by interfering with the repulsive forces that exist between charged polymer molecules in the system. The loss of the 'gel-like' structure results in exposure of the dosage form and drug to the hydrodynamic forces of the dissolution test medium, resulting in an increased exposed surface area with a subsequent faster release rate, as was observed with the VRP dosage forms tested in these studies. The ion-exchange characteristics of Eudragit ${ }^{\circledR}$ RS may enhance the effects of molarity on dissolution of VRP from these dosage forms. Eudragit ${ }^{\circledR} \mathrm{RS}$ contains quaternary ammonium functional groups that dissociate in aqueous media, resulting in chloride ions of the quaternary functional groups exchanging with anionic buffer species during dissolution. At high buffer molarities, the excess buffer ions replace the chloride ions in the polymer, resulting in higher drug release (12).

Drug-polymer interactions in VRP dosage forms decrease as buffer molarity increases, in line with the hypothesis that the ionic bonds between the oppositely charged carbomer and VRP are prevalent. These results are consistent with the findings of Bonferoni et al. (13), who observed a decrease in the percentage of drug bound to polymer with an increase in molarity for lambda carrageenan and diltiazem hydrochloride.

Furthermore, since $\mathrm{KH}_{2} \mathrm{PO}_{4}$ is used in the buffer, there is an increase in the concentration of potassium ions as the buffer molarity is increased. The $\mathrm{K}^{+}$ions would shield the negatively charged carboxylate groups of carbomer, producing a less extended and rigid gel layer structure. These results are in agreement with observations made by Roshdy et al. (14) when measuring tablet gel strength using a cone/plate rheometer. It is more than likely that changes in molarity of the dissolution media impact the structure of the carbomerbased VRP formulations, resulting in a more flexible gel on hydration as indicated by the greater percent drug released.

\section{Effect of Agitation}

The resultant dissolution rate profiles of VRP tablets tested at different agitation rates reveal that the drug release rate

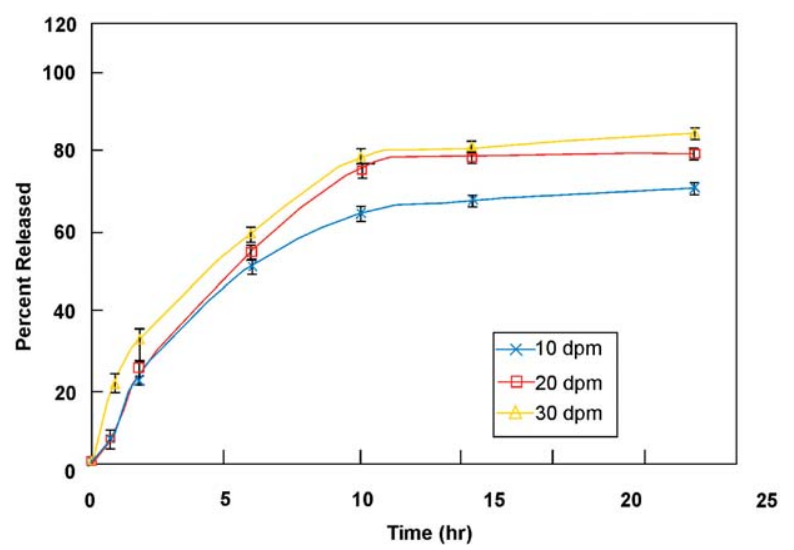

Figure 2. The effect of agitation rate on VRP release from capsules containing hydrophilic matrix tablets (3) reported as mean $\pm S D(n=6)$.

increases with increasing agitation rates (Figure 2). This result was expected, since higher agitation rates produce higher release rates by more effectively reducing the stagnant layer surrounding a tablet, improving sink conditions and promoting tablet erosion (15). As observed from the difference and similarity factors, $\mathrm{f}_{1}$ and $\mathrm{f}_{2}$, summarized in Table 3, there are significant differences between the dissolution profiles of VRP for all comparisons.

In order to elucidate the hydrodynamic conditions thought to be of importance when using USP Apparatus 3 , patterns showing a simplified model of the fluid flow behaviour around tablets in the apparatus are postulated and are depicted in Figures 3 and 4.

A reciprocating rate of $10 \mathrm{dpm}$ would result in a freestream velocity (unimpeded and constant fluid flow), as represented by $V_{0}$, that is lower than that which would predominate at higher agitation rates. The free-stream

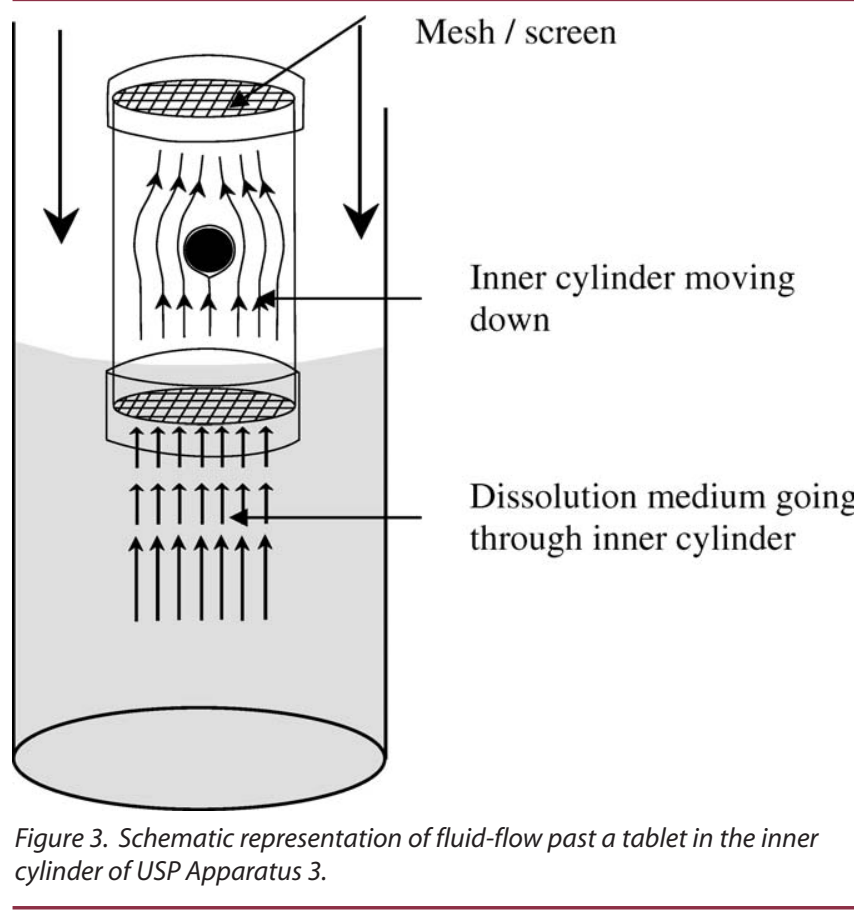


velocity is represented in the figures by lines that indicate the path of fluid around a tablet suspended in a dissolution medium as the inner vessel reciprocates in and out of the dissolution fluid. At lower dpm, it is likely that the flow around the tablet is more smooth and regular with a pseudo-laminar pattern than at higher agitation rates.

At a reciprocation rate of $20 \mathrm{dpm}$, turbulent flow may be generated, which may cause chaotic flow and complex dynamics in the fluids within the dissolution vessel. The fluid currents that are generated are likely to hit the tablet mass at fluctuating velocities in all directions, since the tablets are round or capsule-shaped and present various angles to the direction of fluid flow. Increasing the agitation rate to 30 $\mathrm{dpm}$ increases the intensity of the turbulence in all directions in a fluctuating manner. At higher reciprocation rates, higher fluid flow velocities are likely to weaken the gel structure that immediately forms around a tablet at the commencement of hydration. As the gel layer weakens, the network structure that holds the polymer together slowly breaks down, resulting in a depletion of the layer that ideally acts as the primary retarding region. Therefore, drug is released more rapidly at higher agitation rates, since the surface exposure of the dosage form to the dissolution medium at a specific time is increased.

\section{Effects of Mesh/Screen Size}

It was observed that the dissolution medium in each outer tube covered the upper mesh screen of the inner tube at the lowest point of the reciprocation stroke during dissolution testing. The combination of a fine pore size of the mesh used in the inner tube and the surface tension of the dissolution media created a barrier that prevented air from penetrating the screen and displacing the media in the cylinder, thereby preventing drainage of the test media. This fluid hold-up was only observed when using a low agitation rate of $10 \mathrm{dpm}$. Better drainage occurred at higher agitation rates, since the fluid flowed at a higher free-stream velocity, facilitating air penetration through these openings and subsequently displacing liquids that were retained in the inner tubes.

There have been reports of the effects of dissolution test conditions on drug release when using USP Apparatus 3 (16). In the present study, it was observed that if a $74-\mu \mathrm{m}$ mesh screen was used as the top screen, all cylinders failed to drain completely, and this occurred throughout the entire period of a test run. These observations are consistent with previously reported data (17). When the top screens were replaced with a $177-\mu \mathrm{m}$ mesh screen, better drainage was observed. The larger mesh screen size/pore sizes allow air to penetrate through the mesh openings without difficulty and subsequently displace liquids that may be retained in these inner tubes. The use of a $405-\mu \mathrm{m}$ mesh as a top screen resulted in complete and rapid drainage of all cylinders.

\section{Conclusion}

Sustained-release formulations were successfully prepared using a wet granulation method with carbomer as

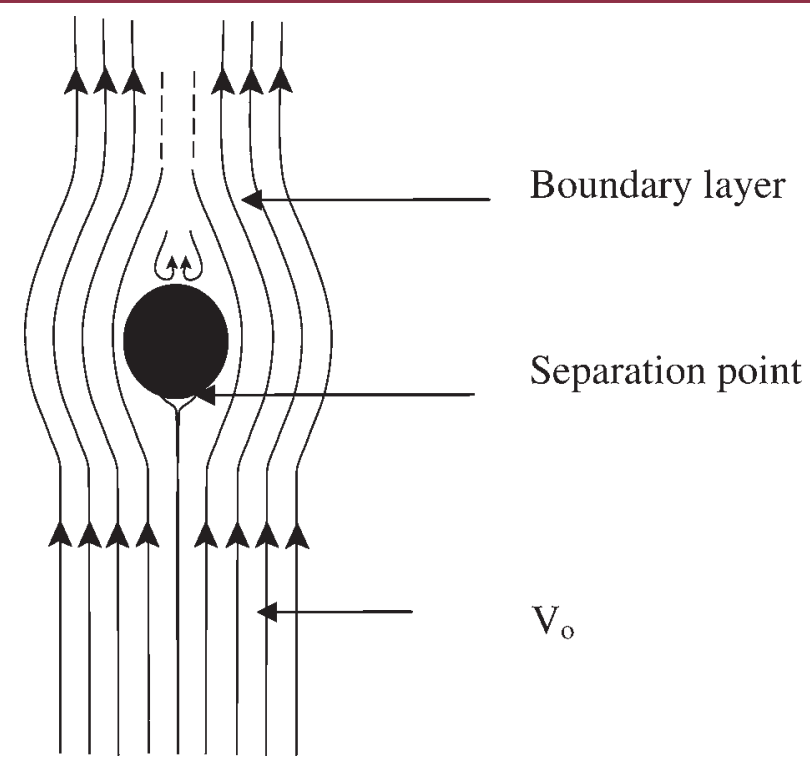

Figure 4. Schematic representation of the free-stream velocity past a tablet when agitated using USP Apparatus 3.

the hydrophilic polymeric carrier. This hydrophilic matrix swelled continuously as dissolution testing progressed. These studies were used in an attempt to understand the influence of different dissolution test conditions on the in vitro release and the fluid flow dynamics at different agitation rates. The choice of agitation intensity and dissolution medium composition is critical for the attainment of appropriate and predictive in vitro results when testing drug release from hydrophilic matrix tablets.

The polymer used showed different in situ gel-forming characteristics. The ability to release entrapped drug in specific media by swelling and cross-linking is related to the swelling capability. Buffer molarity and agitation rate had an impact on the release rate of VRP in the ranges assessed. Furthermore, the choice of appropriate mesh screens and apertures is necessary to facilitate draining of inner tubes when using USP Apparatus 3.

\section{Acknowledgments}

The authors would like to thank the NRF (RBW), Rhodes University (SMMK, RBW). The Dow Chemical Company, Röhm Pharma, Novoen, and Colorcon are gratefully acknowledged for their donation of excipients, and Aspen Pharmacare (Port-Elizabeth, SA) for the verapamil hydrochloride.

\section{References}

1. Kukura, J.; Baxter, J. L.; Muzzio, F. J. Shear distribution and variability in the USP Apparatus 2 under turbulent conditions. Int. J. Pharm. 2004, 279 (1-2), 9-17.

2. Dissolution Testing of Immediate Release Solid Oral Dosage Forms; Guidance for Industry; U.S. Department of Health and Human Services, Food and Drug Administration, U.S. Government Printing Office: Washington, DC, August 1997. 
3. Guo, J. H.; Harcum, W. W.; Skinner, G. W.; Dluzneski, P. R.; Trumbull, D. E. Validation of tablet dissolution method by high performance liquid chromatography. Drug Dev. Ind. Pharm. 2000, 26 (3), 337-342.

4. Siepmann, J.; Lecomte, F.; Bodmeier, R. Diffusion controlled drug delivery systems: calculation of the required composition to achieve desired release profiles. J. Controlled Release 1999, 60 (11), 379-389.

5. Baumgartner, S.; Kristl, J.; Vrecer, F.; Vodopivec, P.; Zorko, B. Optimization of floating matrix tablets and evaluation of their gastric residence time. Int. J. Pharm. 2000, 195 (1-2), 125-135.

6. Lee, B. J.; Ryn, S. G.; Cui, J. H. Formulation and release characteristics of HPMC matrix tablets containing melatonin. Drug Dev. Ind. Pharm. 1999, 25 (4), 493-501.

7. Pillay, V.; Fassihi, R. A new method for dissolution studies of lipid-filled hard shell or softgel capsules. AAPS PharmSci 1998, 1, 83-84.

8. Khamanga, S. M. M. Formulation and Assessment of Verapamil Sustained Release Tablets. M.Sc. Thesis, Rhodes University, Grahamstown, South Africa, 2005.

9. Moore, J. W.; Flanner, H. Mathematical comparison of dissolution profiles. Pharm. Technol. 1996, 20 (6), 64-70.

10. Khamanga, S. M.; Walker, R. B. Evaluation of the Rate of Swelling and Erosion of Verapamil (VRP) SustainedRelease Matrix Tablets. Drug Dev. Ind. Pharm. 2006, 32 (10), 1139-1148.

11. Neau, S. H.; Chow, M. Y.; Durrani, M. J. Fabrication and characterization of extruded and spheronized beads containing Carbopol ${ }^{\circledR} 974$ NF resin. Int .J.Pharm. 1996, 131 (1), 47-55.

12. Bodmeier R.; Guo X.; Sarabia R. E.; Skultety P. F. The influence of buffer species and strength on diltiazem $\mathrm{HCl}$ release from beads coated with the aqueous cationic polymer dispersions, Eudragit RS, RL 30D. Pharm. Res. 1996, 13 (1), 52-56.

13. Bonferoni, M. C.; Rossi, S.; Ferrari, F.; Stavik, E.; PenaRomero, A.; Caramella, C. Factorial analysis of the influence of dissolution medium on drug release from carrageenan-diltiazem complexes. AAPS PharmSciTech 2000, 1 (2) Article 15.

http://www.aapspharmscitech.org/view.asp?art=pt010 215\&pdf=yes (accessed March 2007).

14. Roshdy, M. N.; Schwartz, J. B.; Schnaare, R. L. A novel method for measuring gel strength of controlled release hydrogel tablets using a cone/plate rheometer. Pharm. Dev. Tech. 2001, 6 (1), 107-116.

15. Madden, H.; Butler, J.; Devane, J. Impact of apparatus type and hydrodynamics on the release of a highly soluble drug from a hydrophilic matrix tablet. AAPS PharmSci 1998, 1 (1), 643.

16. Yu, L. X.; Wang, J. T.; Hussain, A. S. Evaluation of USP Apparatus 3 for dissolution testing of immediaterelease products. AAPS PharmSci 2002, 4 (1), 1-5.

17. Rohrs, B. R.; Burch-Clark, D. L.;Witt, M. J.; Stelzer, D. J. USP dissolution Apparatus 3 (reciprocating cylinder): Instrument parameter effects on drug release from sustained-release formulations. J. Pharm. Sci. 1995, 84 (8), 922-926. 